\title{
Cubic Spline Method for a Generalized Black-Scholes Equation
}

\author{
Jian Huang and Zhongdi Cen \\ Institute of Mathematics, Zhejiang Wanli University, Ningbo, Zhejiang 315100, China \\ Correspondence should be addressed to Zhongdi Cen; czdningbo@tom.com \\ Received 10 January 2014; Revised 6 February 2014; Accepted 6 February 2014; Published 6 March 2014 \\ Academic Editor: Kim Meow Liew \\ Copyright (C) 2014 J. Huang and Z. Cen. This is an open access article distributed under the Creative Commons Attribution License, \\ which permits unrestricted use, distribution, and reproduction in any medium, provided the original work is properly cited.
}

\begin{abstract}
We develop a numerical method based on cubic polynomial spline approximations to solve a a generalized Black-Scholes equation. We apply the implicit Euler method for the time discretization and a cubic polynomial spline method for the spatial discretization. We show that the matrix associated with the discrete operator is an M-matrix, which ensures that the scheme is maximum-norm stable. It is proved that the scheme is second-order convergent with respect to the spatial variable. Numerical examples demonstrate the stability, convergence, and robustness of the scheme.
\end{abstract}

\section{Introduction}

An option is a tradable financial contract whose value depends on the value of an underlying asset. The buyer of the contract obtains the right, but not the obligation, to buy or to sell an asset at a specified price on or before a maturity date. A call option provides the right to buy the underlying asset for a certain price, whereas a put option confers the right to sell the underlying asset for a certain price. A European option can only be exercised at the maturity date, while an American option can be exercised at any time prior to its maturity date. Black and Scholes [1] showed that the value of a European option is governed by a second-order parabolic differential equation with respect to the underlying asset price and time, which is known as the Black-Scholes equation. The value of an American option is determined by a linear complementarity problem or as a free boundary value problem involving the Black-Scholes differential operator [2]. It is often necessary to use numerical methods to solve these partial differential equations, as analytic solutions are not generally available.

There are several numerical methods for the valuation of European and American options. The first numerical method for option pricing was the lattice method proposed in Cox et al. [3] and was improved in Hull and White [4], which is equivalent to an explicit time-stepping scheme. Since the Black-Scholes equation with constant or space-independent parameters can be transformed into a diffusion equation, the finite difference methods applied to constant-coefficient heat equations have also been developed (see, e.g., Schwartz
[5], Courtadon [6], Wilmott et al. [2], and Rogers and Talay [7]) for pricing options. Vázquez [8] presented an upwind numerical approach for the Black-Scholes equation. Cen and Le [9-11] presented stable finite difference schemes on a piecewise uniform mesh for pricing European and American options. Wang [12] and Angermann and Wang [13] proposed a fitted finite volume method for the discretization of the Black-Scholes equation. Rambeerich et al. [14] applied the exponential time integration scheme to price options. Other methods, such as meshless approach $[15,16]$, element-free $\mathrm{kp}$-Ritz method $[17,18]$, and element-free Galerkin method [19], also can be used to solve the generalized Black-Scholes equation.

The spline collocation methods are useful methods for solving partial differential equations. Spline solutions have their own advantages. For example, once the solution has been computed, the information between mesh points is available. Numerical methods based on spline collocation methods also have been used to solve option pricing problems. Christara et al. [20] proposed a quadratic spline collocation method to the American option pricing problems. Holtz and Kunoth [21] developed a B-spline-based monotone multigrid method for the valuation of American options. Khabir and Patidar [22] applied a B-spline collocation method to solve the heat equation which is obtained from the Black-Scholes equation by an Euler transformation. Kadalbajoo et al. $[23,24]$ used cubic B-spline collocation methods for the Black-Scholes equation. 
In this paper, we present a numerical method based on cubic polynomial spline to solve a generalized BlackScholes equation. We combine the implicit Euler method for discretizing the time variable with the cubic polynomial spline scheme for discretizing the spatial variable. The matrix associated with the discrete operator is an M-matrix, which ensures that the scheme is maximum-norm stable. We will show that the scheme is second-order convergent with respect to the spatial variable. Numerical results support the theoretical results.

The rest of the paper is organized as follows. In the next section, a generalized Black-Scholes equation is introduced. The temporal semidiscretization is described in Section 3. The spatial discretization is constructed in Section 4. The fully discrete scheme is presented in Section 5. Finally, numerical experiments are provided to support these theoretical results in Section 6.

\section{The Continuous Problem}

We consider the following generalized Black-Scholes equation:

$$
\begin{array}{r}
-\frac{\partial v}{\partial t}-\frac{1}{2} \widehat{\sigma}^{2}(S, t) S^{2} \frac{\partial^{2} v}{\partial S^{2}}-r(t) S \frac{\partial v}{\partial S}+r(t) v=0, \\
(S, t) \in \mathbb{R}^{+} \times(0, T),
\end{array}
$$

equipped with the terminal and boundary conditions

$$
\begin{gathered}
v(S, T)=\max (S-E, 0), \quad x \in \mathbb{R}^{+}, \\
v(0, t)=0, \quad t \in[0, T] .
\end{gathered}
$$

Here, $v(S, t)$ is the value of European call option at asset price $S$ and at time $t, E$ is the exercise price, $T$ is the maturity, $r(t)$ is the risk-free interest rate, and $\widehat{\sigma}(S, t)$ is the volatility function of underlying asset. When $\widehat{\sigma}$ and $r$ are constant functions, it becomes the classical Black-Scholes model. The existence and uniqueness of a classical solution of (1)-(2) are well known (see $[25,26])$.

Note that (1) degenerates when $S$ goes to zero. We transform the Black-Scholes equations (1)-(2) into a nondegenerate partial differential equation by using a log transformation $x=\ln S$

$$
\begin{gathered}
-\frac{\partial u}{\partial t}-\frac{1}{2} \sigma^{2}(x, t) \frac{\partial^{2} u}{\partial x^{2}}-\left(r(t)-\frac{1}{2} \sigma^{2}(x, t)\right) \frac{\partial u}{\partial x} \\
+r(t) u=0, \quad(x, t) \in \mathbb{R} \times(0, T), \\
u(x, T)=\max \left(e^{x}-E, 0\right), \quad x \in \mathbb{R}, \\
u(x, t)=0, \quad \text { as } x \longrightarrow-\infty, t \in[0, T],
\end{gathered}
$$

where $u(x, t)=v(S, t)=v\left(e^{x}, t\right)$ and $\sigma(x, t)=\widehat{\sigma}\left(e^{x}, t\right)$.

For applying the numerical method, we truncate the infinite domain $\mathbb{R} \times(0, T)$ into a truncated domain $\Omega=$ $\left(x_{\min }, x_{\max }\right) \times(0, T)$, where $x_{\min }$ and $x_{\max }$ are chosen properly so that, for practical purposes, they do not affect the option price. Based on Willmott et al.s estimate [2] that the upper bound of the asset price is typically three or four times the strike price, it is reasonable for us to set $x_{\max }=\ln (4 E)$, while, for the lower bound of the asset price, since $-\ln (4 E)$ is negative enough, we take $x_{\min }=-\ln (4 E)$ for convenience in numerical experiments. Therefore, in the remaining of this paper, we will consider the following problem:

$$
\begin{aligned}
& -\frac{\partial u}{\partial t}-\frac{1}{2} \sigma^{2}(x, t) \frac{\partial^{2} u}{\partial x^{2}}-\left(r(t)-\frac{1}{2} \sigma^{2}(x, t)\right) \frac{\partial u}{\partial x} \\
& \quad+r(t) u=0, \quad(x, t) \in \Omega, \\
& u(x, T)=\max \left(e^{x}-E, 0\right), \quad x \in\left(x_{\min }, x_{\max }\right), \\
& u\left(x_{\min }, t\right)=0, \quad t \in[0, T], \\
& u\left(x_{\max }, t\right)=e^{x_{\max }}-E e^{-\int_{t}^{T} r(s) \mathrm{d} s}, \quad t \in[0, T] .
\end{aligned}
$$

Here, the right boundary condition is chosen according to Vázquez [8]. Normally, this truncation of the domain leads to a negligible error in the value of the option [25].

\section{The Temporal Semidiscretization}

To approximate the solution (4), first, we apply the implicit Euler method to discretize the temporal variable. This scheme, on a uniform mesh

$$
\bar{\Omega}^{K}=\left\{t_{j}=j \Delta t, 0 \leq j \leq K, \Delta t=\frac{T}{K}\right\},
$$

reads

$$
\begin{aligned}
& u^{K}=u(x, T)=\max \left(e^{x}-E, 0\right), \\
& \left(I+\Delta t L_{x}\right) u^{j}(x)=u^{j+1}(x), \\
& u^{j}\left(x_{\min }\right)=0, \\
& u^{j}\left(x_{\max }\right)=e^{x_{\max }}-E e^{-\int_{t_{j}}^{T} r(s) \mathrm{d} s}, \\
& \text { for } j=K-1, \ldots, 1,0,
\end{aligned}
$$

where

$$
\begin{aligned}
L_{x} u^{j}(x)= & -\frac{1}{2}\left(\sigma^{j}(x)\right)^{2} \frac{d^{2} u^{j}}{d x^{2}} \\
& -\left(r^{j}-\frac{1}{2}\left(\sigma^{j}(x)\right)^{2}\right) \frac{d u^{j}}{d x} \\
& +r^{j} u^{j}(x),
\end{aligned}
$$

and $u^{j}(x)$ denotes the approximation of the exact solution $u(x, t)$ at the time level $t_{j}$.

Similarly to Kellogg and Tsan [27], we can prove that the differential operator $\left(I+\Delta t L_{x}\right)$ satisfies a maximum principle, and, consequently,

$$
\left\|\left(I+\Delta t L_{x}\right)^{-1}\right\|_{\infty} \leq \frac{1}{1+r \Delta t} .
$$

Hence, we can obtain the following result.

Lemma 1. The temporal semidiscretization scheme (6) is unconditionally stable. 
Estimates for the global error are deduced from appropriate bounds of the local error, where the auxiliary problem

$$
\begin{gathered}
\left(I+\Delta t L_{x}\right) \widehat{u}^{j}=u\left(x, t_{j+1}\right), \\
\widehat{u}^{j}\left(x_{\min }\right)=0, \\
\widehat{u}^{j}\left(x_{\max }\right)=e^{x_{\max }}-E e^{-\int_{t_{j}}^{T} r(s) \mathrm{d} s},
\end{gathered}
$$

is introduced to define the local error.

Lemma 2 (local error estimate). The local error associated with the method (9), defined by $e^{j}=u\left(x, t_{j}\right)-\widehat{u}^{j}(x)$, satisfies

$$
\left\|e^{j}\right\|_{\infty}=O\left((\Delta t)^{2}\right) .
$$

Proof. Using Taylor expansion, we have

$$
\begin{aligned}
-\frac{u\left(x, t_{j+1}\right)-u\left(x, t_{j}\right)}{\Delta t} & =-u_{t}\left(x, t_{j}\right)+O(\Delta t) \\
& =-L_{x} u\left(x, t_{j}\right)+O(\Delta t),
\end{aligned}
$$

for $1 \leq j<K$. From (9) and (11), it is straightforward to show that the local error is the solution of the problem

$$
\begin{gathered}
\left(I+\Delta t L_{x}\right) e^{j}=O\left((\Delta t)^{2}\right), \\
e^{j}\left(x_{\min }\right)=e^{j}\left(x_{\max }\right)=0,
\end{gathered}
$$

and, therefore, the result follows from the maximum principle for the operator $\left(I+\Delta t L_{x}\right)$.

Lemma 3 (global error estimate). The global error associated with the implicit Euler method (6), given by $E^{j}=u\left(x, t_{j}\right)-$ $u^{j}(x)$, satisfies

$$
E=\sup _{j \leq K}\left\|E^{j}\right\|_{\infty}=O(\Delta t),
$$

and, therefore, the temporal semidiscretization scheme is a firstorder convergent scheme.

Proof. The global truncation error at time $t_{j}$ can be decomposed as

$$
\begin{aligned}
E^{j} & =u\left(x, t_{j}\right)-u^{j}(x) \\
& =\left(u\left(x, t_{j}\right)-\widehat{u}^{j}(x)\right)+\left(\widehat{u}^{j}(x)-u^{j}(x)\right) .
\end{aligned}
$$

By relations (6) and (9), we have

$$
\left(I+\Delta t L_{x}\right)\left(\widehat{u}^{j}(x)-u^{j}(x)\right)=u\left(x, t_{j+1}\right)-u^{j+1}(x) .
$$

Applying the maximum principle for the operator $\left(I+\Delta t L_{x}\right)$, we can obtain

$$
\left\|\widehat{u}^{j}(x)-u^{j}(x)\right\|_{\infty} \leq \frac{1}{1+r \Delta t}\left\|u\left(x, t_{j+1}\right)-u^{j+1}(x)\right\|_{\infty} .
$$

Thus, from (14)-(16), we have

$$
\begin{aligned}
\left\|E^{j}\right\|_{\infty} & \leq C\left(\left\|e^{j}\right\|_{\infty}+\left\|e^{j+1}\right\|_{\infty}+\cdots+\left\|e^{K}\right\|_{\infty}\right) \\
& \leq C \Delta t,
\end{aligned}
$$

for $0 \leq j \leq K$, where $C$ is a positive constant independent from $\Delta t$.

\section{The Spatial Discretization}

For the approximate solution of the semidiscretization problem (6), the spatial discretization is performed on a uniform mesh

$$
\bar{\Omega}^{N}=\left\{x_{i}=i h, 0 \leq i \leq N, h=\frac{\left(x_{\max }-x_{\min }\right)}{N}\right\},
$$

for the computational domain $\left[x_{\min }, x_{\max }\right]$. Thus, at each time point $t_{j}$, we apply a cubic spline scheme on the above uniform mesh $\Omega^{N}$ to approximate problem (6).

Let $S_{\Delta}^{j}(x)$ be the approximate solution of the exact solution $u^{j}(x)$ of the boundary value problem (6) at the $j$ th time level. At each subinterval $\left[x_{i}, x_{i+1}\right]$, the cubic spline function $S_{\Delta}^{j}(x)$ has the following form:

$$
\begin{aligned}
S_{\Delta}^{j}(x)= & a_{i}^{j}+b_{i}^{j}\left(x-x_{i}\right)+c_{i}^{j}\left(x-x_{i}\right)^{2} \\
& +d_{i}^{j}\left(x-x_{i}\right)^{3}, \quad i=0,1, \ldots, N-1,
\end{aligned}
$$

where $a_{i}^{j}, b_{i}^{j}, c_{i}^{j}$, and $d_{i}^{j}$ are constants. Using the notation $U_{i}^{j}$ for approximation of $u^{j}(x)$ at mesh points $x_{i}$ and $S_{\Delta}^{j}\left(x_{i}\right)=$ $U_{i}^{j}, S_{\Delta}^{j}\left(x_{i+1}\right)=U_{i+1}^{j}$ as interpolatory constraints. From algebraic manipulation, we can obtain

$$
\begin{aligned}
& a_{i}^{j}=U_{i}^{j}, \\
& b_{i}^{j}=\frac{U_{i+1}^{j}-U_{i}^{j}}{h}-\frac{h\left(M_{i+1}^{j}-M_{i}^{j}\right)}{6}, \\
& c_{i}^{j}=\frac{M_{i}^{j}}{2}, \\
& d_{i}^{j}=\frac{M_{i+1}^{j}-M_{i}^{j}}{6 h},
\end{aligned}
$$

where $M_{i}^{j}=\left(S_{\Delta}^{j}\right)^{\prime \prime}\left(x_{i}\right)$. Using the continuity of the first derivative at mesh point $x_{i}$, we get the following equation:

$$
M_{i+1}^{j}+4 M_{i}^{j}+M_{i-1}^{j}=\frac{6}{h^{2}}\left(U_{i+1}^{j}-2 U_{i}^{j}+U_{i-1}^{j}\right) .
$$

Substituting

$$
M_{i}^{j}=\frac{-2 u_{i}^{j+1}-\Delta t\left(2 r^{j}-\left(\sigma_{i}^{j}\right)^{2}\right) u_{x, i}^{j}+2\left(1+\Delta t r^{j}\right) u_{i}^{j}}{\Delta t\left(\sigma_{i}^{j}\right)^{2}}
$$

in (21) and using the following approximations for first-order derivative of $u^{j}$

$$
\begin{aligned}
u_{x, i}^{j} & \simeq \frac{U_{i+1}^{j}-U_{i-1}^{j}}{2 h}, \\
u_{x, i+1}^{j} & \simeq \frac{3 U_{i+1}^{j}-4 U_{i}^{j}+U_{i-1}^{j}}{2 h}, \\
u_{x, i-1}^{j} & \simeq \frac{-U_{i+1}^{j}+4 U_{i}^{j}-3 U_{i-1}^{j}}{2 h},
\end{aligned}
$$


we get the following spline difference scheme:

$$
\begin{aligned}
L^{N} U_{i}^{j}= & \frac{2 h^{2}}{\left(\sigma_{i-1}^{j}\right)^{2}} U_{i-1}^{j+1}+\frac{8 h^{2}}{\left(\sigma_{i}^{j}\right)^{2}} U_{i}^{j+1} \\
& +\frac{2 h^{2}}{\left(\sigma_{i+1}^{j}\right)^{2}} U_{i+1}^{j+1}, \quad i=1,2, \ldots, N-1, \\
U_{0}^{j}= & 0, \quad U_{N}^{j}=e^{x_{\max }}-E e^{-\int_{t_{j}}^{T} r(s) \mathrm{d} s},
\end{aligned}
$$

where

$$
\begin{aligned}
& L^{N} U_{i}^{j}=\left[-6 \Delta t-\frac{1}{2} h \Delta t p_{i+1}^{j}+2 h \Delta t p_{i}^{j}\right. \\
& \left.+\frac{3}{2} h \Delta t p_{i-1}^{j}+\frac{2 h^{2}\left(1+\Delta t r^{j}\right)}{\left(\sigma_{i-1}^{j}\right)^{2}}\right] U_{i-1}^{j} \\
& +\left[12 \Delta t+2 h \Delta t p_{i+1}^{j}-2 h \Delta t p_{i-1}^{j}\right. \\
& \left.+\frac{8 h^{2}\left(1+\Delta t r^{j}\right)}{\left(\sigma_{i}^{j}\right)^{2}}\right] U_{i}^{j} \\
& +\left[-6 \Delta t-\frac{3}{2} h \Delta t p_{i+1}^{j}-2 h \Delta t p_{i}^{j}\right. \\
& \left.+\frac{1}{2} h \Delta t p_{i-1}^{j}+\frac{2 h^{2}\left(1+\Delta t r^{j}\right)}{\left(\sigma_{i+1}^{j}\right)^{2}}\right] U_{i+1}^{j}
\end{aligned}
$$

and $p_{i}^{j}=2 r^{j} /\left(\sigma_{i}^{j}\right)^{2}-1$.

It is easy to see that the matrix associated with the discrete operator $L^{N}$ is an M-matrix for sufficiently small $h$. Hence, the following discrete maximum principle holds true.

Lemma 4 (discrete maximum principle). For sufficiently small $h$, the operator $L^{N}$ defined by (25) on the uniform mesh $\Omega^{N}$ satisfies a discrete maximum principle; that is, if $w$ is a mesh function that satisfies $w_{0} \geq 0, w_{N} \geq 0$, and $L^{N} w_{i} \geq$ $0 \quad(1 \leq i<N)$, then $w_{i} \geq 0$, for all $i$.

From the above lemma, we can conclude that the spatial discretization scheme (24) is maximum-norm stable.

To prove the convergence of the spline difference scheme, we discretize the auxiliary problem (9) and obtain

$$
\begin{aligned}
& L^{N} \widehat{U}_{i}^{j}= \frac{2 h^{2}}{\left(\sigma_{i-1}^{j}\right)^{2}} u\left(x_{i-1}, t_{j+1}\right)+\frac{8 h^{2}}{\left(\sigma_{i}^{j}\right)^{2}} u\left(x_{i}, t_{j+1}\right) \\
&+\frac{2 h^{2}}{\left(\sigma_{i+1}^{j}\right)^{2}} u\left(x_{i+1}, t_{j+1}\right), \quad i=1,2, \ldots, N-1, \\
& \widehat{U}_{0}^{j}=0, \quad \widehat{U}_{N}^{j}=e^{x_{\max }}-E e^{-\int_{t_{j}}^{T} r(s) \mathrm{d} s} .
\end{aligned}
$$

Lemma 5. Let $\widehat{u}^{j}(x)$ be the solution of (9) and $\left\{\widehat{U}_{i}^{j}\right\}$ be the solution of (26). Then, we have the following error estimate:

$$
\left|\widehat{u}^{j}\left(x_{i}\right)-\widehat{U}_{i}^{j}\right| \leq C h^{2} \Delta t, \quad 0 \leq i \leq N .
$$

Proof. We use a Taylor expansion at $x=x_{i}$ to obtain the following local truncation error estimate:

$$
\begin{aligned}
& \left|L^{N}\left(\widehat{u}_{i}^{j}-\widehat{U}_{i}^{j}\right)\right|=\mid L^{N} \widehat{u}_{i}^{j}-\frac{2 h^{2}}{\left(\sigma_{i-1}^{j}\right)^{2}} u\left(x_{i-1}, t_{j+1}\right) \\
& -\frac{8 h^{2}}{\left(\sigma_{i}^{j}\right)^{2}} u\left(x_{i}, t_{j+1}\right) \\
& -\frac{2 h^{2}}{\left(\sigma_{i+1}^{j}\right)^{2}} u\left(x_{i+1}, t_{j+1}\right) \\
& =\mid L^{N} \widehat{u}_{i}^{j}-\frac{2 h^{2}}{\left(\sigma_{i-1}^{j}\right)^{2}}\left(I+\Delta t L_{x}\right) \widehat{u}_{i-1}^{j} \\
& -\frac{8 h^{2}}{\left(\sigma_{i}^{j}\right)^{2}}\left(I+\Delta t L_{x}\right) \widehat{u}_{i}^{j} \\
& -\frac{2 h^{2}}{\left(\sigma_{i+1}^{j}\right)^{2}}\left(I+\Delta t L_{x}\right) \widehat{u}_{i+1}^{j} \\
& \leq \frac{1}{3} h^{4} \Delta t\left(\left|p_{i-1}^{j}\right|+2\left|p_{i}^{j}\right|+\left|p_{i+1}^{j}\right|\right) \\
& \times\left|\frac{d^{3} \widehat{u}^{j}}{d x^{3}}\left(\xi_{i}\right)\right| \\
& \leq C h^{4} \Delta t \text {. }
\end{aligned}
$$

Hence, using the discrete maximum principle (Lemma 4) for the discrete operator $L^{N}$, we have

$$
\left|\widehat{u}^{j}\left(x_{i}\right)-\widehat{U}_{i}^{j}\right| \leq C h^{2} \Delta t, \quad 0 \leq i \leq N,
$$

which completes the proof.

\section{The Fully Discrete Scheme}

Combining the time semidiscretization scheme (6) with the spatial discretization scheme (24), we can obtain the following fully discretization scheme:

$$
\begin{gathered}
U_{i}^{K}=\max \left(e^{x}-E, 0\right), \\
L^{N} U_{i}^{j}=\frac{2 h^{2}}{\left(\sigma_{i-1}^{j}\right)^{2}} U_{i-1}^{j+1}+\frac{8 h^{2}}{\left(\sigma_{i}^{j}\right)^{2}} U_{i}^{j+1} \\
+\frac{2 h^{2}}{\left(\sigma_{i+1}^{j}\right)^{2}} U_{i+1}^{j+1}, \quad 1 \leq i<N, \\
U_{0}^{j}=0, \quad U_{N}^{j}=e^{x_{\max }}-E e^{-\int_{t_{j}}^{T} r(s) \mathrm{d} s}, \\
\text { for } j=K-1, \ldots, 1,0,
\end{gathered}
$$


where the discrete operators $L^{N}$ are described in Section 4 and $U_{i}^{j}$ is the fully discrete approximation to the exact solution of (4) at the mesh point $\left(x_{i}, t_{j}\right)$.

Now, we can get the main result for our difference scheme.

Theorem 6. Let $u(x, t)$ be the exact solution of (4) and let $U$ be the discrete solution of the fully discrete scheme (30). Then, the global error of our difference scheme satisfies

$$
\left|u\left(x_{i}, t_{j}\right)-U_{i}^{j}\right| \leq C\left(h^{2}+\Delta t\right), \quad 0 \leq i \leq N, 0 \leq j \leq K,
$$

where $C$ is a positive constant independent of $h$ and $\Delta t$.

Proof. The global error at the time $t_{j}$ can be decomposed in the form

$$
\begin{aligned}
\left|u\left(x_{i}, t_{j}\right)-U_{i}^{j}\right| \leq & \left|u\left(x_{i}, t_{j}\right)-\widehat{u}^{j}\left(x_{i}\right)\right| \\
& +\left|\widehat{u}^{j}\left(x_{i}\right)-\widehat{U}_{i}^{j}\right|+\left|\widehat{U}_{i}^{j}-U_{i}^{j}\right| .
\end{aligned}
$$

From Lemmas 2 and 5, we can obtain

$$
\left|u\left(x_{i}, t_{j}\right)-U_{i}^{j}\right| \leq C \Delta t\left(h^{2}+\Delta t\right)+\left|\widehat{U}_{i}^{j}-U_{i}^{j}\right| .
$$

Further, it is easy to see that $\widehat{U}^{j}-U^{j}$ can be written as the solution of one step of (30) with zero boundary conditions and $u\left(x, t_{j+1}\right)-U^{j+1}$ as the final value. Applying the stability of the discrete scheme (Lemma 4), we have

$$
\left|\widehat{U}_{i}^{j}-U_{i}^{j}\right| \leq C\left\|u\left(x, t_{j+1}\right)-U^{j+1}\right\|_{\infty} .
$$

Then, from (33) and (34), a recurrence relation for the global errors follows, and, from it, the result of Theorem 6 can be obtained immediately.

\section{Numerical Experiments}

In this section, we present some numerical results to examine the performance and convergence of the cubic spline method. Errors and convergence rates for the numerical scheme are presented for three examples.

Example 1. European call option with parameters: $\sigma=$ $0.4, r=0.08, T=1, E=1, x_{\min }=-\ln (4 E)$, and $x_{\max }=$ $\ln (4 E)$ : in this case, the analytical solution is

$$
v(S, \tau)=S N\left(d_{1}\right)-E e^{-r(T-\tau)} N\left(d_{2}\right),
$$

where

$$
\begin{gathered}
N(x)=\frac{1}{\sqrt{2 \pi}} \int_{-\infty}^{x} e^{-y^{2} / 2} \mathrm{~d} y, \\
d_{1}(S, \tau)=\frac{\ln (S / E)+\left(r+(1 / 2) \sigma^{2}\right)(T-\tau)}{\sigma \sqrt{T-\tau}}, \\
d_{2}(S, \tau)=d_{1}(S, \tau)-\sigma \sqrt{T-\tau} .
\end{gathered}
$$

The maximum error is given in Table 1 . The analytical and numerical solution profiles are given in Figure 1.
TABLE 1: Numerical results for Example 1.

\begin{tabular}{lccc}
\hline$N$ & $K$ & Error $e^{N, K}$ & Rate $r^{N, K}$ \\
\hline 8 & 4 & $1.2469 e-02$ & - \\
16 & 16 & $2.9318 e-03$ & 2.089 \\
32 & 64 & $7.2583 e-04$ & 2.014 \\
64 & 256 & $1.8143 e-04$ & 2.000 \\
128 & 1024 & $4.5346 e-05$ & 2.000 \\
\hline
\end{tabular}

TABLE 2: Numerical results for Example 2.

\begin{tabular}{lccc}
\hline$N$ & $K$ & Error $e^{N, K}$ & Rate $r^{N, K}$ \\
\hline 8 & 4 & $1.8366 e-02$ & - \\
16 & 16 & $6.7729 e-03$ & 1.439 \\
32 & 64 & $1.4290 e-03$ & 2.245 \\
64 & 256 & $3.6112 e-04$ & 1.985 \\
128 & 1024 & $8.9871 e-05$ & 2.007 \\
\hline
\end{tabular}

TABLE 3: Numerical results for Example 3.

\begin{tabular}{lccc}
\hline$N$ & $K$ & Error $e^{N, K}$ & Rate $r^{N, K}$ \\
\hline 8 & 4 & $1.8364 e-02$ & - \\
16 & 16 & $6.7715 e-03$ & 1.439 \\
32 & 64 & $1.4277 e-03$ & 2.246 \\
64 & 256 & $3.5967 e-04$ & 1.989 \\
128 & 1024 & $8.8418 e-05$ & 2.024 \\
\hline
\end{tabular}

Example 2. European call option with parameters: $\sigma=$ $0.1, r=0.06, T=1, E=1, x_{\min }=-\ln (4 E)$ and $x_{\max }=$ $\ln (4 E)$ : as in Example 1, in this case, the analytical solution is known.

The maximum error is given in Table 1 . The analytical and numerical solution profiles are given in Figure 2.

Example 3. European call option with parameters: $\sigma(S, \tau)=$ $0.15(0.5+2 \tau)\left((S / 100-1.2)^{2} /\left((S / 100)^{2}+1.44\right)\right), r=0.06, T=$ $1, E=1, x_{\min }=-\ln (4 E)$, and $x_{\max }=\ln (4 E)$ : here, the volatility function $\sigma(S, \tau)$ is the same as the one given in Toivanen [28] and Kadalbajoo et al. [23, 24].

In this case, the exact solution is not known. We use the approximated solution of $N=2048$ and $K=4096$ as the exact solution. We present the error estimates for different $N$ and $K$. Let $U^{2048,4096}$ denote "the exact solution." We measure the accuracy in the discrete maximum norm

$$
e^{N, K}=\max _{i, j}\left|U_{i j}^{N, K}-U_{i, j}^{2048,4096}\right|
$$

and the convergence rate

$$
R^{N, K}=\log _{2}\left(\frac{e^{N, K}}{e^{2 N, 4 K}}\right) .
$$

The error estimates and convergence rates are listed in Table 3. The analytical and numerical solution profiles are given in Figure 3.

From Figures (1)-(3), it is seen that the numerical solutions by our method are nonoscillatory. From Tables 1, 


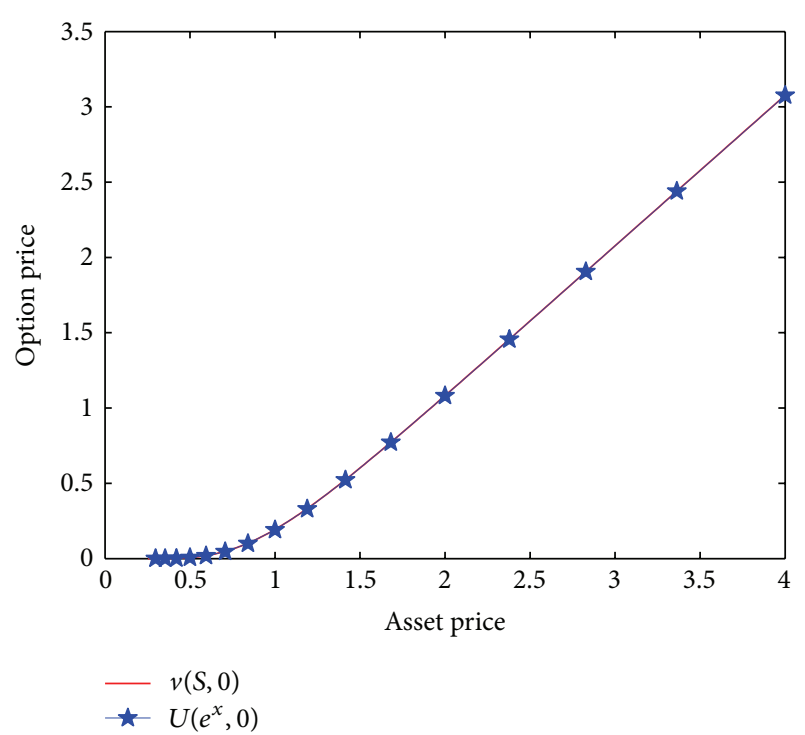

Figure 1: Option value at $t=0$ for Example 1.

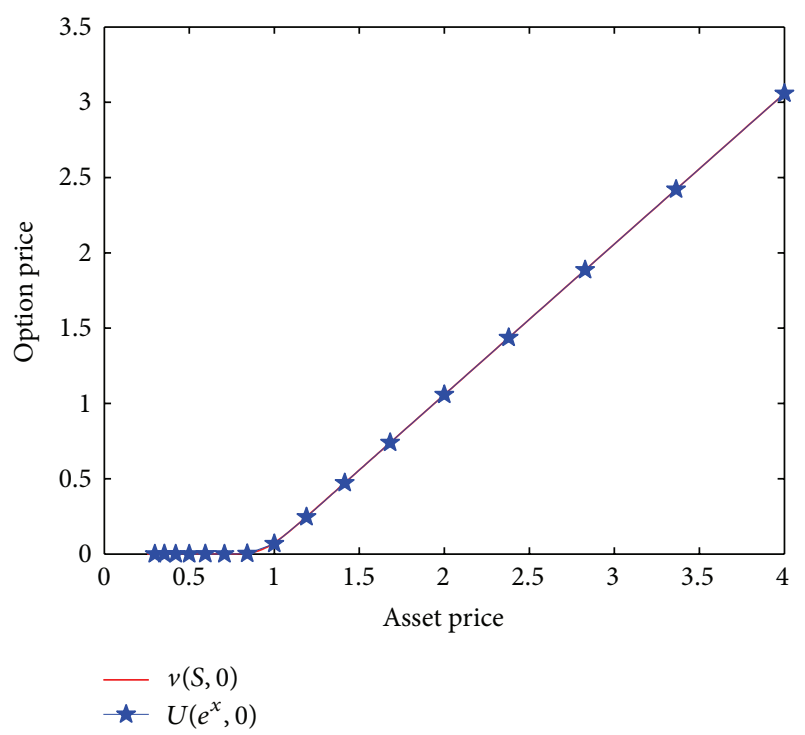

Figure 2: Option value at $t=0$ for Example 2.

2 , and 3 , we see that $e^{N, K} / e^{2 N, 4 K}$ is close to 4 , which supports the convergence estimate of Theorem 6 . They indicate that the theoretical results are fairly sharp.

\section{Conflict of Interests}

The authors declare that there is no conflict of interests regarding the publication of this paper.

\section{Acknowledgments}

The authors would like to thank the anonymous referee for several suggestions for the improvement of this paper. The work was supported by the National Natural Science Foundation (Grant no. 11201430) of China, Ningbo Municipal

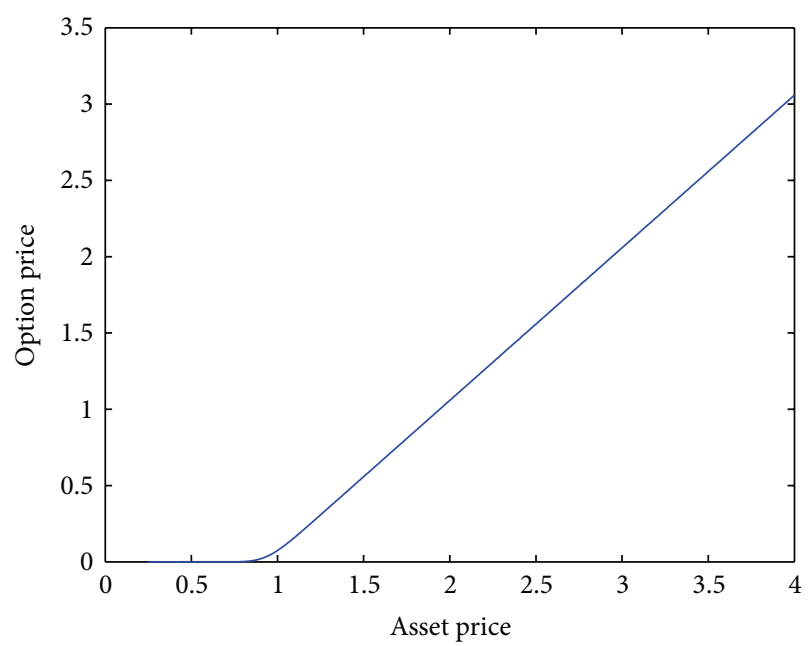

Figure 3: Option value at $t=0$ for Example 3.

Natural Science Foundation, Projects in Science and Technique of Ningbo Municipal (Grant no. 2012B82003) of China, and Key Research Center of Philosophy and Social Science of Zhejiang Province-Modern Port Service Industry and Creative Culture Research Center.

\section{References}

[1] F. Black and M. S. Scholes, "The pricing of options and corporate liabilities," Journal of Political Economy, vol. 81, no. 3, pp. 637654, 1973.

[2] P. Wilmott, J. Dewynne, and S. Howison, Option Pricing: Mathematical Models and Computation, Oxford Financial Press, Oxford, UK, 1993.

[3] J. C. Cox, S. Ross, and M. Rubinstein, "Option pricing: a simplified approach," A Simplified Approach, vol. 7, no. 3, pp. 229264, 1979.

[4] J. C. Hull and A. White, "The use of control variate technique in option pricing," Journal of Financial and Quantitative Analysis, vol. 23, no. 3, pp. 237-251, 1988.

[5] E. S. Schwartz, "The valuation of warrants: implementing a new approach," Journal of Financial Economics, vol. 4, no. 1, pp. 7993, 1977.

[6] G. Courtadon, "A more accurate finite difference approximation for the valuation of options," Journal of Financial and Quantitative Analysis, vol. 17, no. 5, pp. 697-703, 1982.

[7] L. C. G. Rogers and D. Talay, Numercial Methods in Finance, Cambridge University Press, Cambridge, UK, 1997.

[8] C. Vázquez, "An upwind numerical approach for an American and European option pricing model," Applied Mathematics and Computation, vol. 97, no. 2-3, pp. 273-286, 1998.

[9] Z. Cen and A. Le, "A robust finite difference scheme for pricing American put options with singularity-separating method," Numerical Algorithms, vol. 53, no. 4, pp. 497-510, 2010.

[10] Z. Cen and A. Le, "A robust and accurate finite difference method for a generalized Black-Scholes equation," Journal of Computational and Applied Mathematics, vol. 235, no. 13, pp. 37282733, 2011.

[11] Z. Cen, A. Le, and A. Xu, "A second-order difference scheme for the penalized black-scholes equation governing American put 
option pricing," Computational Economics, vol. 40, no. 1, pp. 4962, 2012.

[12] S. Wang, "A novel fitted finite volume method for the Black-Scholes equation governing option pricing," IMA Journal of Numerical Analysis, vol. 24, no. 4, pp. 699-720, 2004.

[13] L. Angermann and S. Wang, "Convergence of a fitted finite volume method for the penalized Black-Scholes equation governing European and American option pricing," Numerische Mathematik, vol. 106, no. 1, pp. 1-40, 2007.

[14] N. Rambeerich, D. Y. Tangman, A. Gopaul, and M. Bhuruth, "Exponential time integration for fast finite element solutions of some financial engineering problems," Journal of Computational and Applied Mathematics, vol. 224, no. 2, pp. 668-678, 2009.

[15] K. M. Liew, Z. X. Lei, J. L. Yu, and L. W. Zhang, "Postbuckling of carbon nanotube-reinforced functionally graded cylindrical panels under axial compression using a meshless approach," Computer Methods in Applied Mechanics and Engineering, vol. 268, pp. 1-17, 2014.

[16] L. W. Zhang, P. Zhu, and K. M. Liew, "Thermal buckling of functionally graded plates using a local Kriging meshless method," Composite Structures, vol. 108, pp. 472-492, 2014.

[17] R. J. Cheng, L. W. Zhang, and K. M. Liew, "Modeling of biological population problems using the element-free kp-Ritz method," Applied Mathematics and Computation, vol. 227, pp. 274290, 2014.

[18] L. W. Zhang, Z. X. Lei, K. M. Liew, and J. L. Yu, "Static and dynamic of carbon nanotube reinforced functionally graded cylindrical panels," Composite Structures, vol. 111, pp. 205-212, 2014.

[19] L. W. Zhang, Y. J. Deng, and K. M. Liew, "An improved elementfree Galerkin method for numerical modeling of the biological population problems," Engineering Analysis with Boundary Elements, vol. 40, pp. 181-188, 2014.

[20] C. C. Christara, T. Chen, and D. M. Dang, "Quadratic spline collocation for one-dimensional linear parabolic partial differential equations," Numerical Algorithms, vol. 53, no. 4, pp. 511553,2010 .

[21] M. Holtz and A. Kunoth, "B-spline-based monotone multigrid methods," SIAM Journal on Numerical Analysis, vol. 45, no. 3, pp. 1175-1199, 2007.

[22] M. H. M. Khabir and K. C. Patidar, "Spline approximation method to solve an option pricing problem," Journal of Difference Equations and Applications, vol. 18, no. 11, pp. 1801-1816, 2012.

[23] M. K. Kadalbajoo and L. P. Tripathi, "A robust nonuniform Bspline collocation method for solving the generalized BlackScholes equation," IMA Journal of Numerical Analysis, vol. 34, no. 1, pp. 252-278, 2014.

[24] M. K. Kadalbajoo, L. P. Tripathi, and A. Kumar, "A cubic B-spline collocation method for a numerical solution of the generalized Black-Scholes equation," Mathematical and Computer Modelling, vol. 55, no. 3-4, pp. 1483-1505, 2012.

[25] R. Kangro and R. Nicolaides, "Far field boundary conditions for Black-Scholes equations," SIAM Journal on Numerical Analysis, vol. 38 , no. 4, pp. 1357-1368, 2000.

[26] O. A. Ladyzenskaja, V. A. Solonnikov, and N. N. Ural'ceva, Linear and Quasilinear Equations of Parabolic Type, American Mathematical Society, Providence, RI, USA, 1968.

[27] R. B. Kellogg and A. Tsan, "Analysis of some difference approximations for a singular perturbation problem without turning points," Mathematics of Computation, vol. 32, no. 144, pp. 10251039, 1978.
[28] J. Toivanen, "Numerical valuation of European and American options under Kou's jump-diffusion model," SIAM Journal on Scientific Computing, vol. 30, no. 4, pp. 1949-1970, 2008. 


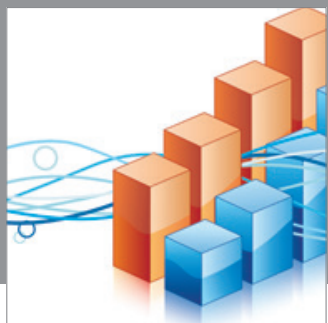

Advances in

Operations Research

mansans

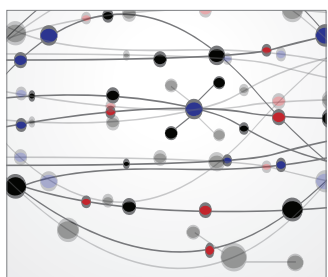

The Scientific World Journal
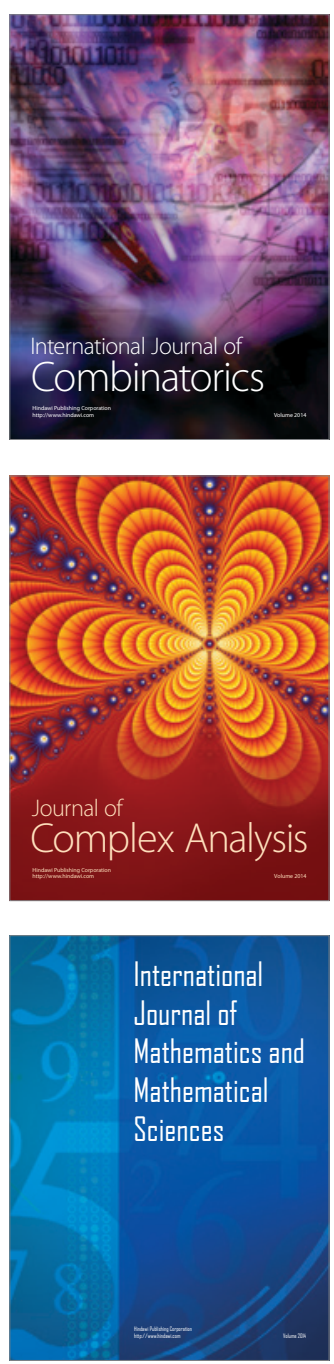
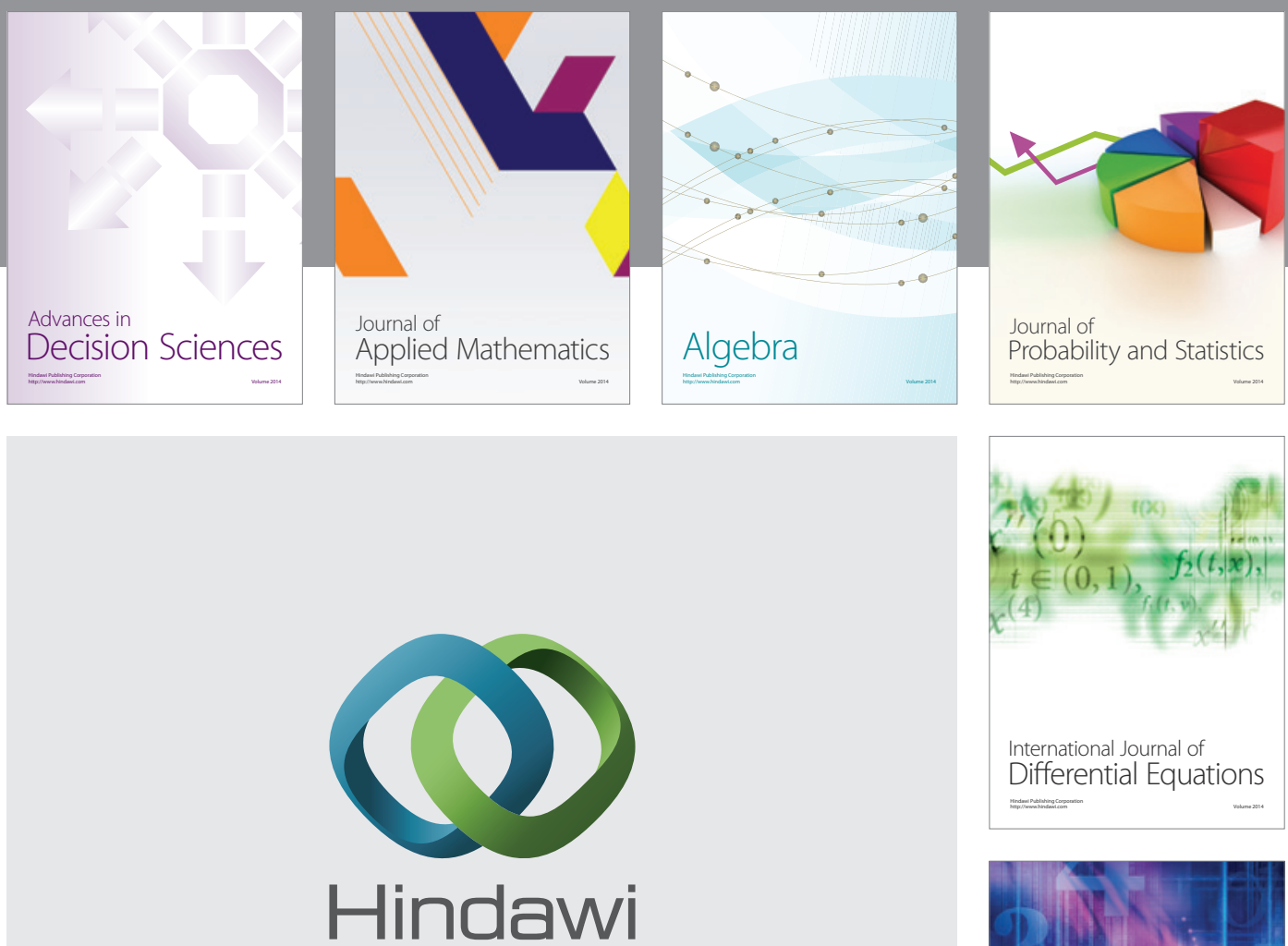

Submit your manuscripts at http://www.hindawi.com
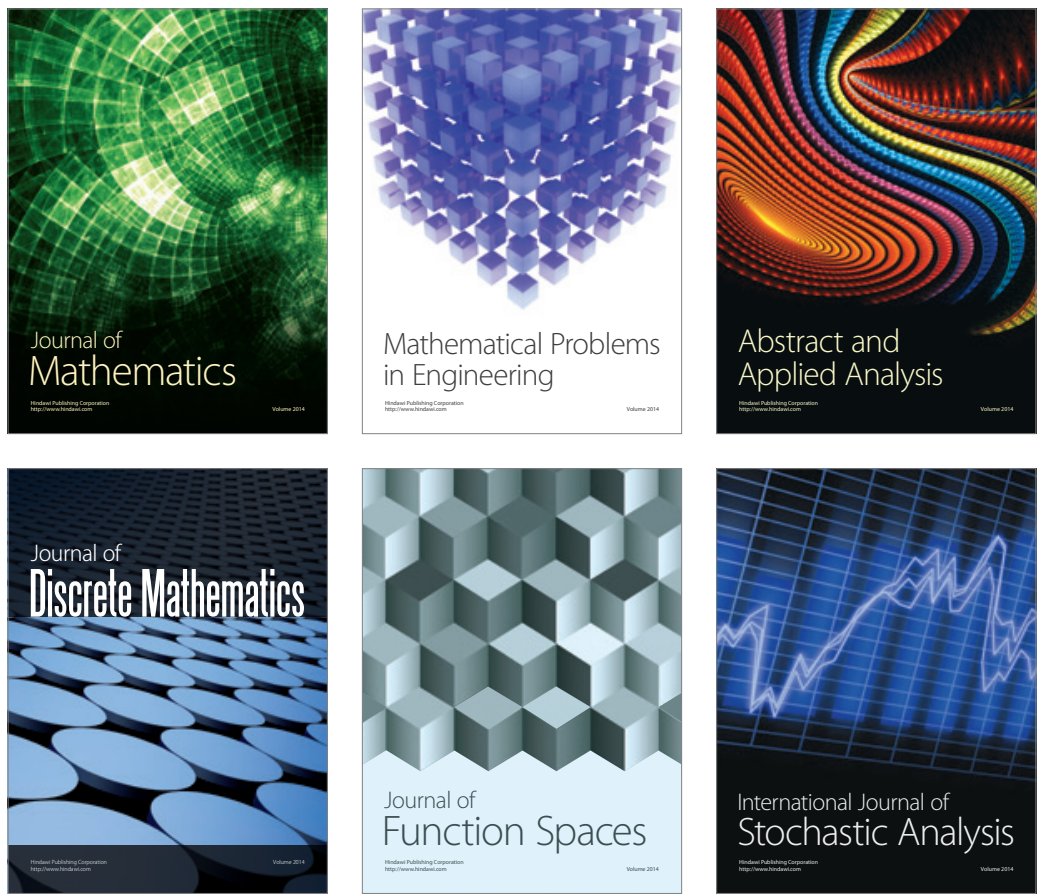

Journal of

Function Spaces

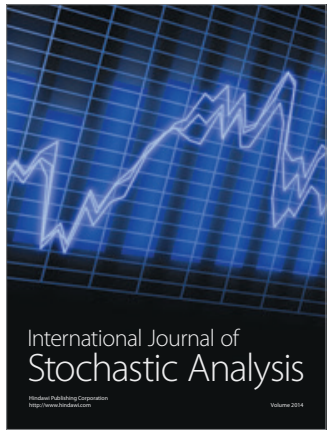

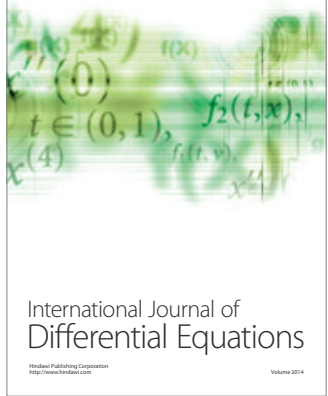
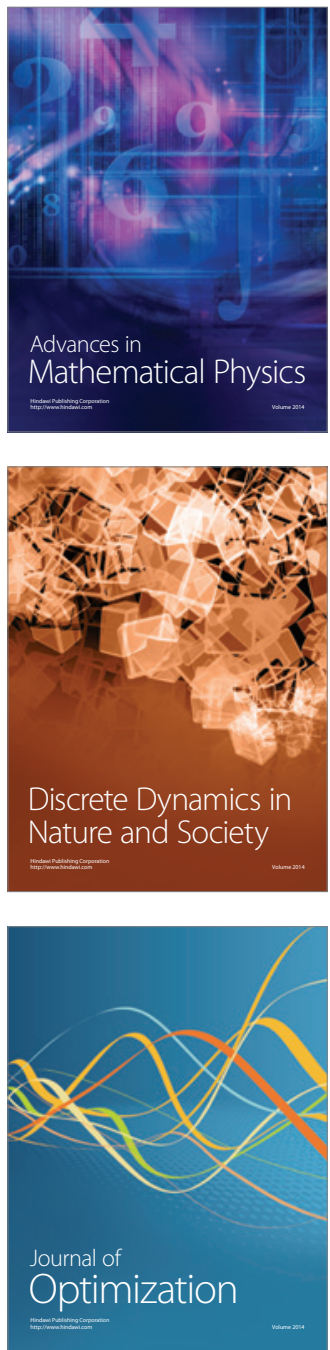